\title{
Single cell RNA sequencing identifies an early monocyte gene signature in acute respiratory distress syndrome
}

\author{
Yale Jiang, ${ }^{1,2}$ Brian R. Rosborough, ${ }^{3}$ Jie Chen, ${ }^{3}$ Sudipta Das, ${ }^{3}$ Georgios D. Kitsios, ${ }^{3}$ Bryan J. McVerry, ${ }^{3}$ \\ Rama K. Mallampalli, ${ }^{4}$ Janet S. Lee, ${ }^{3}$ Anuradha Ray, ${ }^{3,5}$ Wei Chen, ${ }^{1}$ and Prabir Ray, \\ 'Division of Pulmonary, Allergy and Critical Care Medicine, Department of Medicine, University of Pittsburgh, Pittsburgh, \\ Pennsylvania, USA. ${ }^{2}$ School of Medicine, Tsinghua University, Beijing, China. ${ }^{3}$ Division of Pulmonary, Allergy and Critical \\ Care Medicine, Department of Medicine, and ${ }^{4}$ Pulmonary, Critical Care, and Sleep Medicine, The Ohio State University \\ Wexner Medical Center, Davis Heart Lung Research Institute, Columbus, Ohio. ${ }^{5}$ Department of Immunology, University of \\ Pittsburgh, Pittsburgh, Pennsylvania, USA.
}

\begin{abstract}
Acute respiratory distress syndrome (ARDS) results from overwhelming pulmonary inflammation. Prior bulk RNA sequencing provided limited insights into ARDS pathogenesis. We used single cell RNA sequencing to probe ARDS at a higher resolution. PBMCs of patients with pneumonia and sepsis with early ARDS were compared with those of sepsis patients who did not develop ARDS. Monocyte clusters from ARDS patients revealed multiple distinguishing characteristics in comparison with monocytes from patients without ARDS, including downregulation of SOCS3 expression, accompanied by a proinflammatory signature with upregulation of multiple type I IFN-induced genes, especially in CD16+ cells. To generate an ARDS risk score, we identified upregulation of 29 genes in the monocytes of these patients, and 17 showed a similar profile in cells of patients in independent cohorts. Monocytes had increased expression of RAB11A, known to inhibit neutrophil efferocytosis; ATP2B1, a calcium pump that exports $\mathrm{Ca}^{2+}$ implicated in endothelial barrier disruption; and SPARC, associated with processing of procollagen to collagen. These data show that monocytes of ARDS patients upregulate expression of genes not just restricted to those associated with inflammation. Together, our findings identify molecules that are likely involved in ARDS pathogenesis that may inform biomarker and therapeutic development.
\end{abstract}

Authorship note: YJ, BRR, and JC contributed equally to this work.

Conflict of interest: The authors have declared that no conflict of interest exists.

Copyright: (c) 2020, American Society for Clinical Investigation.

Submitted: December 12, 2019

Accepted: June 3, 2020

Published: June 18, 2020.

Reference information: JCl Insight. 2020;5(13):e135678.

https://doi.org/10.1172/jci.

insight.135678.

\section{Introduction}

The acute respiratory distress syndrome (ARDS) is a clinical syndrome that is often life threatening and affects 3 million people worldwide, accounting for up to $10 \%$ of intensive care unit (ICU) admissions (1). Its clinical manifestations include acute hypoxemia, noncardiogenic pulmonary edema, and reduced lung compliance (increase in stiffness). ARDS can precipitate as a result of a variety of pulmonary insults (pneumonia typically resulting from bacterial or viral infections, gastric aspiration) or nonpulmonary insults (sepsis, trauma, pancreatitis). ARDS carries high mortality (30\%-40\%), and survivors experience long-term sequelae of the syndrome, including pulmonary function limitations and sustained neurocognitive deficiencies (2)

Despite decades of intense research and multiple clinical trials, treatment options remain limited, relying on supportive care with invasive mechanical ventilation and efforts to reverse the underlying etiology (3-5). Targeted interventions require a deeper understanding of the mechanisms that induce ARDS. Furthermore, ARDS develops rapidly after the initial insult, and since it is not possible to assess lung injury directly, and no consensus is yet reached regarding biomarkers that can be included in the definition of ARDS, indirect clinical criteria are relied upon for a diagnosis of ARDS (6).

During the initial exudative phase of lung injury in ARDS patients, innate immune cells, including neutrophils and monocytes, are recruited to the alveolar airspaces and cause damage to epithelial and endothelial barriers, resulting in accumulation of proteinaceous edema fluid in the alveoli and interstitium (6-9). During lung injury, resident alveolar macrophages secrete proinflammatory cytokines that aid in the recruitment of circulating neutrophils and mononuclear cells to the lung, causing sustenance of inflammation and tissue 
injury (10). The inflammatory phase of ARDS is followed by a fibroproliferative phase characterized by resolution of inflammation and deposition of collagen $(7,11,12)$. Although the inflammatory cascade resulting in acute lung injury is relatively understood in mouse models, mechanistic understanding of ARDS in humans remains limited.

Prior studies have used the microarray platform to observe patients with sepsis compared with those with sepsis and acute lung injury/ARDS to identify transcripts associated with ARDS. In one study, an 8-gene signature was able to distinguish between patients with sepsis and patients with sepsis with acute lung injury (13). Further investigation found elevated RNA of IL-1 superfamily cytokines with a statistically significant increase in IL-1 $\beta$ and a trend toward an increase in IL-18 when observing patients with ARDS and sepsis compared with patients lacking ARDS (14). IL-18 has been associated with increased mortality in sepsis-induced ARDS (15). Expression of genes related to neutrophil function was also found to be elevated in patients with ARDS when compared with patients with sepsis alone (16). Taken together, these studies suggest that differential gene expression in PBMCs may be associated with ARDS development and underlie mechanisms of inflammatory injury to the lungs in patients with sepsis. However, microarray data are limited in their ability to resolve activated transcription programs at the cellular level. Single cell RNA sequencing (scRNA-seq) overcomes this limitation by unbiased sequencing of transcripts in individual cells and has the potential to provide deeper insights into the mechanisms of ARDS (17).

In this study, we performed scRNA-seq to identify early transcriptomic signatures associated with the development of ARDS in patients with pneumonia-associated direct lung injury. PBMCs were isolated from immunocompetent patients within 24 hours of intubation to test the hypothesis that early transcriptomic changes are identifiable by scRNA-seq that can differentiate between patients who develop ARDS versus those who do not, secondary to pneumonia. In our attempt to identify molecular signatures that are associated with diffuse alveolar injury and capillary leak, ARDS patients selected for this analysis were compared with patients requiring mechanical ventilatory support for sepsis and pneumonia but did not meet clinical criteria for ARDS (18). Our study has identified previously unrecognized differences in gene expression in NK cells and monocyte clusters of patients with ARDS as compared with those who did not develop ARDS that may help in future biomarker and therapeutic development.

\section{Results}

Subject characteristics. Peripheral blood samples were collected from 4 patients at risk for ARDS with pneumonia and sepsis (sepsis only) and 3 patients who had advanced to ARDS with sepsis and pneumonia (sepsis+ARDS) within 24 hours of initiation of mechanical ventilation (Figure 1A). Subjects ranged in age from 36-78 years, with median age of 51 years, as summarized in Table 1. A diagnosis of ARDS was made retrospectively according to the Berlin definition of exposure to a known ARDS risk factor within 1 week and development of bilateral opacities on chest radiograph that are not fully explained by cardiogenic pulmonary edema (18). Compared with subjects with ARDS, the sepsis-only group had a higher proportion of males. One ARDS patient tested positive for influenza, but the pathogen was not identified in the other 2 cases. Ventilator settings at the time of enrollment are included (Table 1).

Cluster identification. Cell types were identified in an unbiased manner after batch correction and normalization. Main clusters commonly observed in PBMCs, as well as subpopulations within monocytes and T cells, were identified (Figure 1, B and C; Supplemental Table 1; Supplemental Table 2; Supplemental Figure 1; and Supplemental Figure 2, A and B; supplemental material available online with this article; https://doi.org/10.1172/jci.insight.135678DS1). The Mono/B cluster was suspected to be composed of multiplets of monocytes and B cells, while the Mono/T cluster with relatively high proportion of mitochondrial genes indicated poor quality (Supplemental Figure 1). CD14+ monocytes were further clustered into 4 subpopulations. Two cell clusters could not be identified and were excluded from further analysis. We found a significant decrease in the number of NK cells, which may be due to recruitment of these clusters to the focus of inflammation or differential expression of specific genes, as discussed below. The proportion of B cells was elevated in ARDS (Figure 1D). Genes used to identify cell clusters were conserved in both patient groups (Figure 1E).

Differentially expressed genes in cells from sepsis-only versus sepsis $+A R D S$ subjects. We identified 53 differentially expressed genes (DEGs) between sepsis-only and sepsis+ARDS subjects in all cells (Bonferroni-adjusted $P<0.01$, absolute value of average log fold change $[\operatorname{logFC}]>0.1$, Supplemental Table 3). Among them, several IFN-related genes were upregulated in sepsis+ARDS compared with sepsis-only patients. 
A

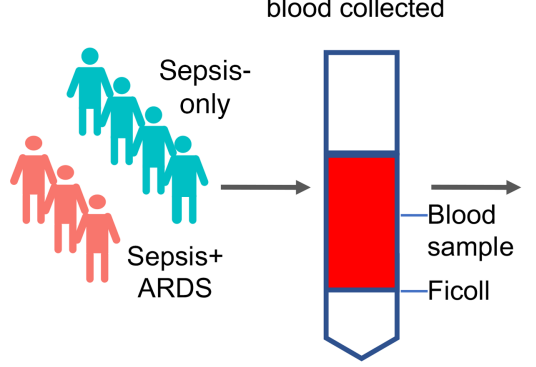

PBMC

isolated

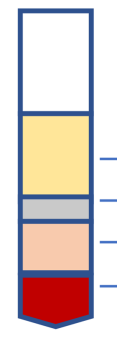

PBMC cryopreserved
PBMC

thawed
B

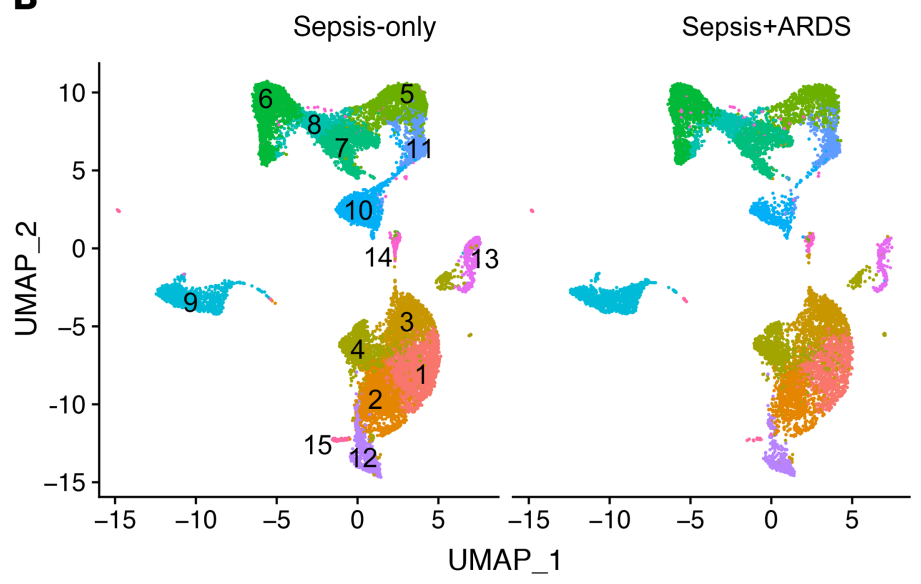

C

- CD14Mono-1 (1)

- CD14Mono-2(2)

- CD14Mono-3(3)

- CD14Mono-4 (4)

- CD8T (5)

- CD4T-1 (6)

- CD4T-2 (7)

- CD4T-3 (8)

- B (9)

- NK (10)

- NKT (11)

- CD16Mono (12)

- Mk (13)

- Mono/T (14)

Mono/B (15)

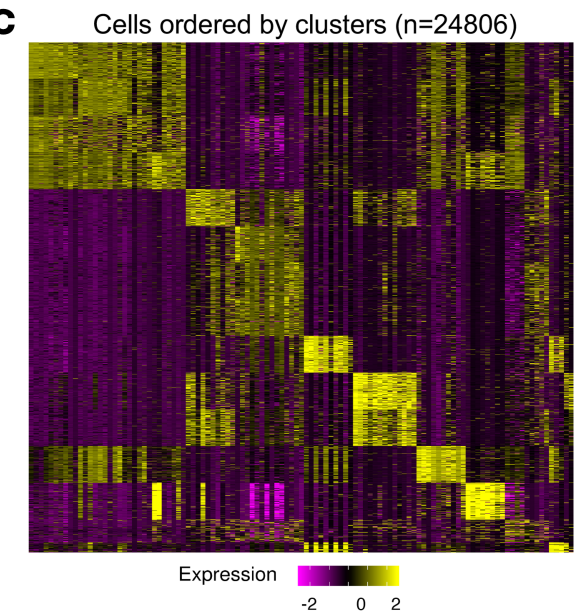

D

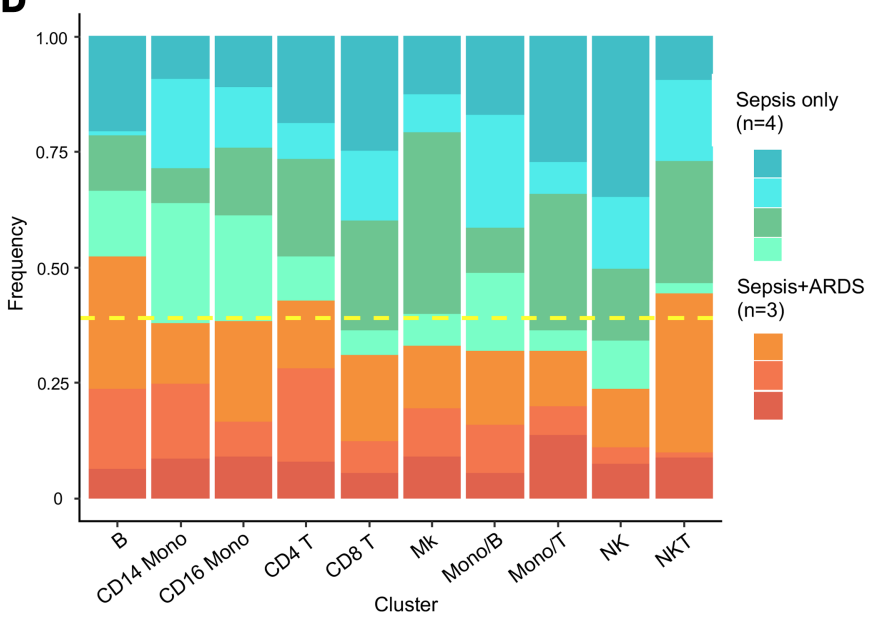

\section{E}

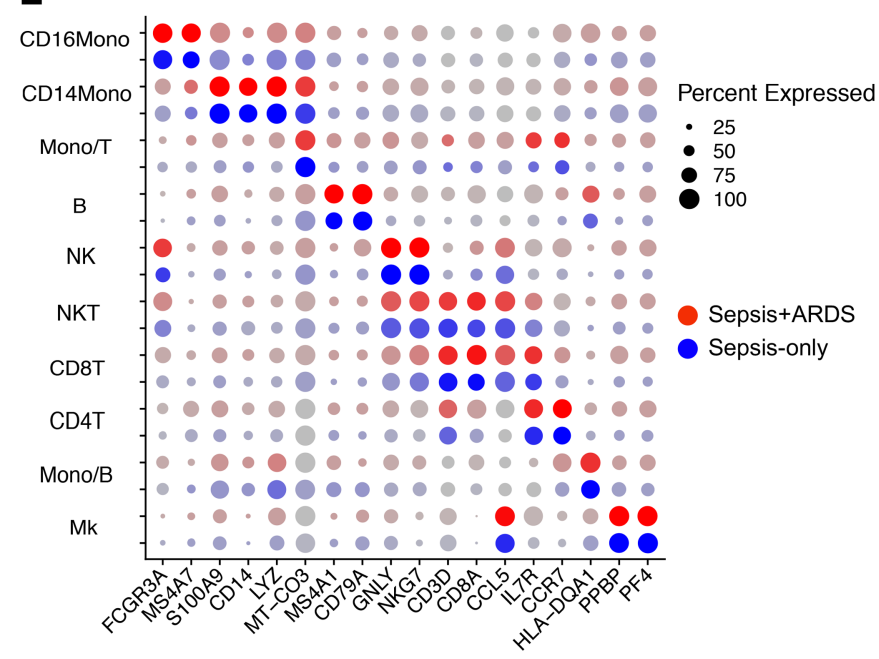

Figure 1. scRNA-seq analysis reveals the cellular composition of PBMCs in sepsis-only and sepsis+ARDS patients. (A) The schematic illustrates the experimental design and work flow. (B) PBMCs were analyzed from sepsis only $(n=4)$ and sepsis+ARDS $(n=3)$ patients and projected with uniform manifold approximation and projection (UMAP) plots with colors and the number in parenthesis indicating the identified cell cluster. (C) The heatmap depicts the marker genes corresponding to each cluster identified in B. (D) The frequency of each cell type is depicted in the columns. Each patient is represented by an individual color in a shade of blue/green (sepsis-only) or orange/red (sepsis+ARDS). The yellow dashed line represents an equal frequency between the 2 groups, since there are 4 sepsis-only patients and 3 sepsis+ARDS patients. (E) The plot shows identification of marker genes for each cell cluster, with the size of the dot corresponding to the percentage of cells within the cell population expressing the gene. The brightness of the color represents the average expression level across all cells within the cluster. Blue and red dots indicate sepsis-only and sepsis+ARDS patients, respectively. CD14Mono, CD14+ monocyte; CD8T, CD8 ${ }^{+} \mathrm{T}$ cell; CD4T, CD4+ T cell; B, B cell; NK, NK cell; NKT, NK T cell; CD16Mono, CD16+ monocytes; Mk, megakaryocyte.

In a recently reported study that employed bulk RNA-seq, one of these genes - IFI44L, an important paralog of the gene IFN-induced protein 44, an IFN stimulated gene (ISG) - was found to be differentially expressed between ARDS and ARDS-hematopoietic stem cell transplanted patients (19). Other intriguing genes included $H L A-D Q B 1$, a member of the HLA complex, and NAMPT, a regulator of intracellular NAD 
Table 1. Patient characteristics

\begin{tabular}{|c|c|c|c|c|c|c|c|c|c|c|}
\hline Sample & Age & Sex & Sepsis & ARDS & Pneumonia & $\begin{array}{l}\text { Pneumonia } \\
\text { pathogen }\end{array}$ & SOFA & $\begin{array}{c}\text { Tidal volume } \\
\text { (cc/kg) }\end{array}$ & PEEP & $\begin{array}{l}\text { Plateau } \\
\text { pressure }\end{array}$ \\
\hline Sepsis_1 & 65 & Female & + & - & + & $\begin{array}{c}\text { Klebsiella } \\
\text { pneumoniae }\end{array}$ & 6 & 7.09 & 5 & 16 \\
\hline Sepsis_2 & 62 & Male & + & - & + & $\begin{array}{c}\text { Klebsiella } \\
\text { oxytoca, MSSA }\end{array}$ & 7 & 7.28 & 14 & 20 \\
\hline Sepsis_3 & 51 & Male & + & - & + & $\begin{array}{l}\text { Enterobacter } \\
\text { species }\end{array}$ & 6 & 7.16 & 5 & 14 \\
\hline Sepsis_4 & 78 & Male & + & - & + & $\begin{array}{c}\text { Enterobacter } \\
\text { aerogenes, E. } \\
\text { coli, MRSA }\end{array}$ & 9 & 7.1 & 5 & 16 \\
\hline ARDS_1 & 73 & Female & + & + & + & Influenza & 7 & 8.05 & 8 & 21 \\
\hline ARDS_2 & 51 & Female & + & + & + & $N / A$ & 4 & 7.32 & 8 & 22 \\
\hline ARDS_3 & 36 & Female & + & + & + & $N / A$ & 4 & 5.84 & 18 & 27 \\
\hline $\begin{array}{l}\text { Patient den } \\
\text { SOFA, sequ } \\
\text { Staphyloco }\end{array}$ & $\begin{array}{l}\text { ograp } \\
\text { ntial o }\end{array}$ & $\begin{array}{l}\text { ics, pneum } \\
\text { rgan failure } \\
\text { eus. }\end{array}$ & $\begin{array}{l}\text { nonia pat } \\
\text { e assessi }\end{array}$ & $\begin{array}{l}\text { hogen, a } \\
\text { nent; PE }\end{array}$ & $\begin{array}{l}\text { and ventilator } \\
\text { EP, positive e }\end{array}$ & $\begin{array}{l}\text { r settings at the t } \\
\text { end-expiratory pre }\end{array}$ & $\begin{array}{l}\text { ime of e } \\
\text { essure. } 1\end{array}$ & MSSA, Methicill & $\begin{array}{l}\text { lown. } \\
\text { n-sensit }\end{array}$ & ive \\
\hline
\end{tabular}

pool and cellular metabolism (20). AREG, which encodes amphiregulin, was among the downregulated genes. Downregulation of $A R E G$ induces epithelial cell apoptosis in LPS-induced lung injury in mice (21).

Canonical signaling pathways are activated in NK cells in ARDS. In agreement with a reduced frequency of NK cells shown in Figure 1D, the t-Distributed Stochastic Neighbor Embedding (t-SNE) profiles in Figure 2A show reduction in NK cell numbers in ARDS patients. Figure 2B shows that overall gene expression was also reduced in the NK cells, which was especially true for specific genes such as PTGDS (encodes prostaglandin D2 synthase). Interestingly, genetic deletion of the isoform of prostaglandin D2 synthase found in hematopoietic cells resulted in increased inflammation at the outset in a mouse model of peritonitis and also impaired resolution of inflammation (22). Furthermore, prostaglandin D2 has been shown to inhibit NK cell secretion of type 1 cytokines (23). Expression of the IFN-inducible genes, IFI44L and IFITM3, was upregulated in NK cells of ARDS patients, while, interestingly, expression of IFNGRI was undetectable (Figure 2C). IFNGR1 is ubiquitously expressed by all cell types, and its downregulation may prevent proinflammatory responses to IFN- $\gamma$, as recently observed in human monocytes (24). Pathway enrichment analysis using the 102 suppressed genes (Bonferroni-corrected $P<0.01, \operatorname{logFC}<-0.1$ ) revealed pathways inhibited in ARDS patients. Altogether, 29 pathways were found to be significantly downregulated (Bonferroni-corrected $P<0.01$, Supplemental Table 4). The most significantly enriched pathways in each biological process group are shown in Figure 2D. In ARDS patients, the combined downregulation of genes that negatively regulate $\mathrm{NF}-\mathrm{\kappa B}$ activation, negatively regulate mitogen-activated protein (MAP) kinases, and also negatively regulate serine/threonine protein kinases suggests an increase in activation status of NK cells in the context of ARDS.

Type I IFN-associated pathways and transcription factors are upregulated in the monocytes of ARDS patients. Monocytes are heterogeneous and dynamic in circulation. We identified 4 clusters of CD14 $4^{+}$monocytes and 1 cluster of $\mathrm{CD} 16^{+}$monocytes (Figure 3, A and B). The first cluster of CD14 $4^{+}$monocytes showed high expression of CES1, also known as monocyte esterase. CD74, along with HLA family genes, were markers for the second cluster. The third cluster expressed higher levels of CTSD, which encodes lysosomal protease cathepsin $\mathrm{D}$, while the last cluster of monocytes had high expression of platelet basic protein precursor gene (PPBP) (Figure 3C). These 4 clusters of $\mathrm{CD}_{14}{ }^{+}$monocytes could represent various stages of differentiation of monocytes, which may have functional consequences in ARDS. The proportion of each of the monocyte subsets in the 2 patient groups was not significantly different, which is shown in Supplemental Figure 3.

Pathways enriched in the top DEGs in each monocyte subpopulation in patients with sepsis+ARDS relative to those in patients with sepsis only were examined (Bonferroni-adjusted $P<0.01, \operatorname{logFC}>0.1$; Supplemental Table 5). Pathways corresponding to upregulated genes are illustrated in Figure 3D, where each node denotes a biological process while colors of the nodes distinguish the 5 monocyte subpopulations. Both $\mathrm{CD} 16^{+}$monocytes and $\mathrm{CD} 14^{+}$monocytes from the $C E S 1$ (cluster 1) and CD74 (cluster 2) clusters 
A

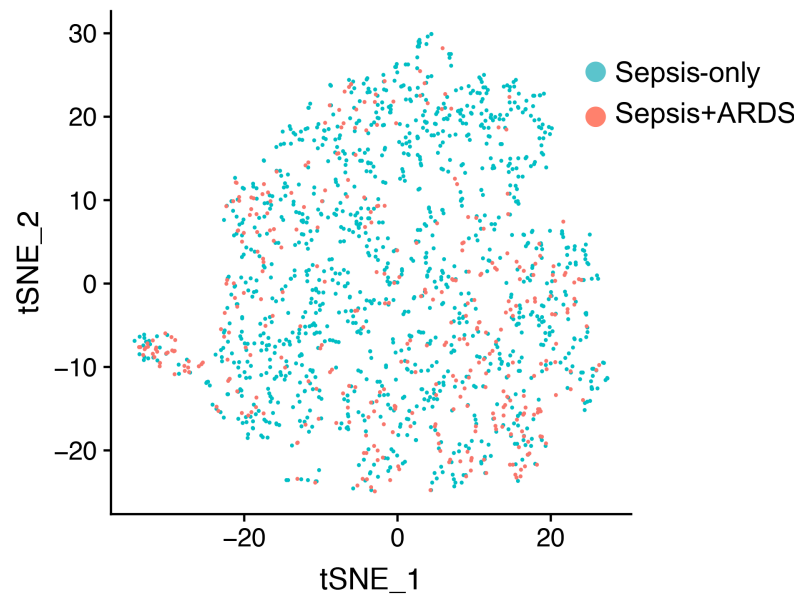

C

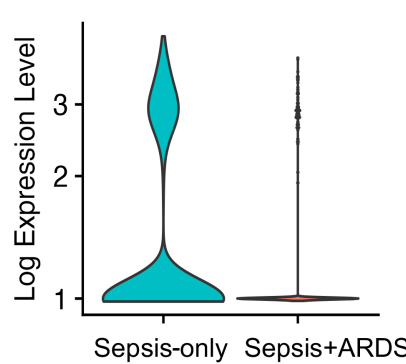

Sepsis-only Sepsis+ARDS
IFITM3

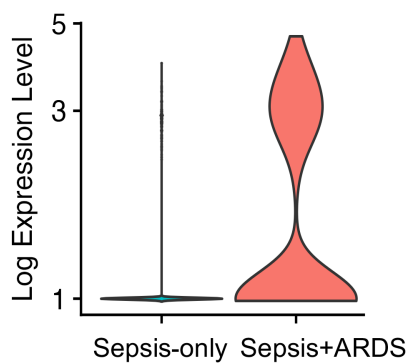

B

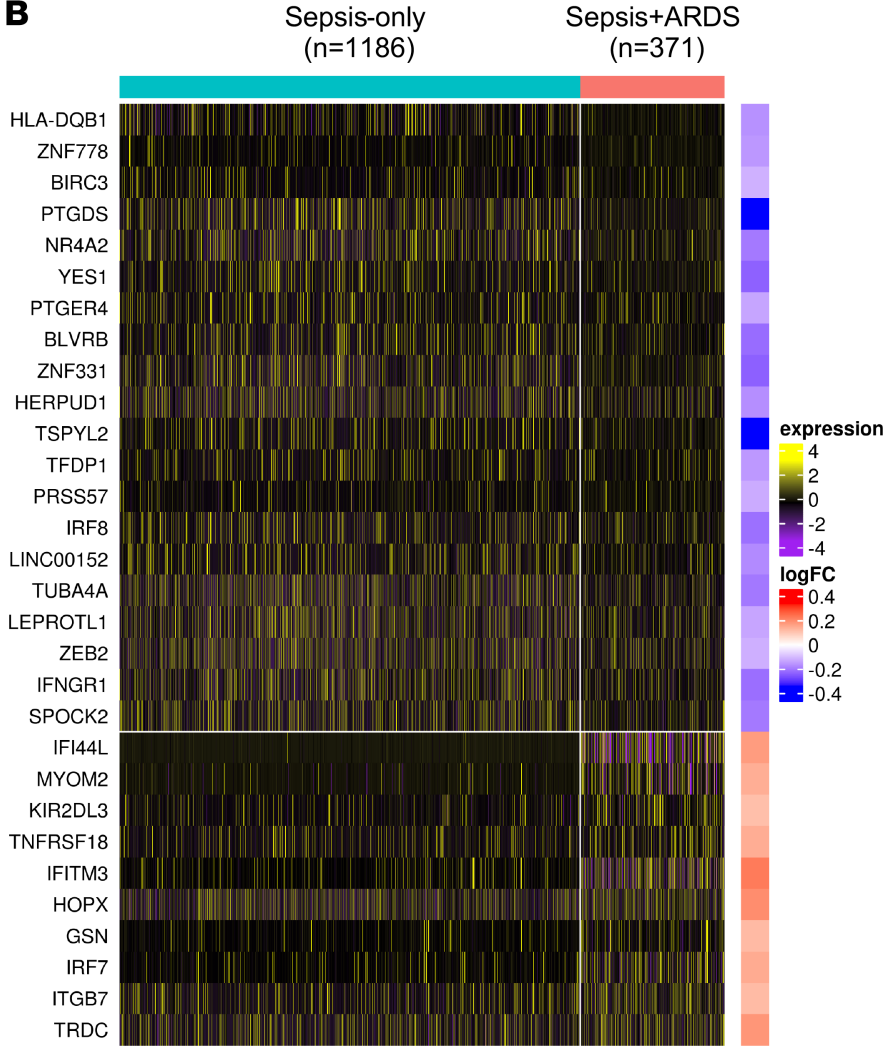

D

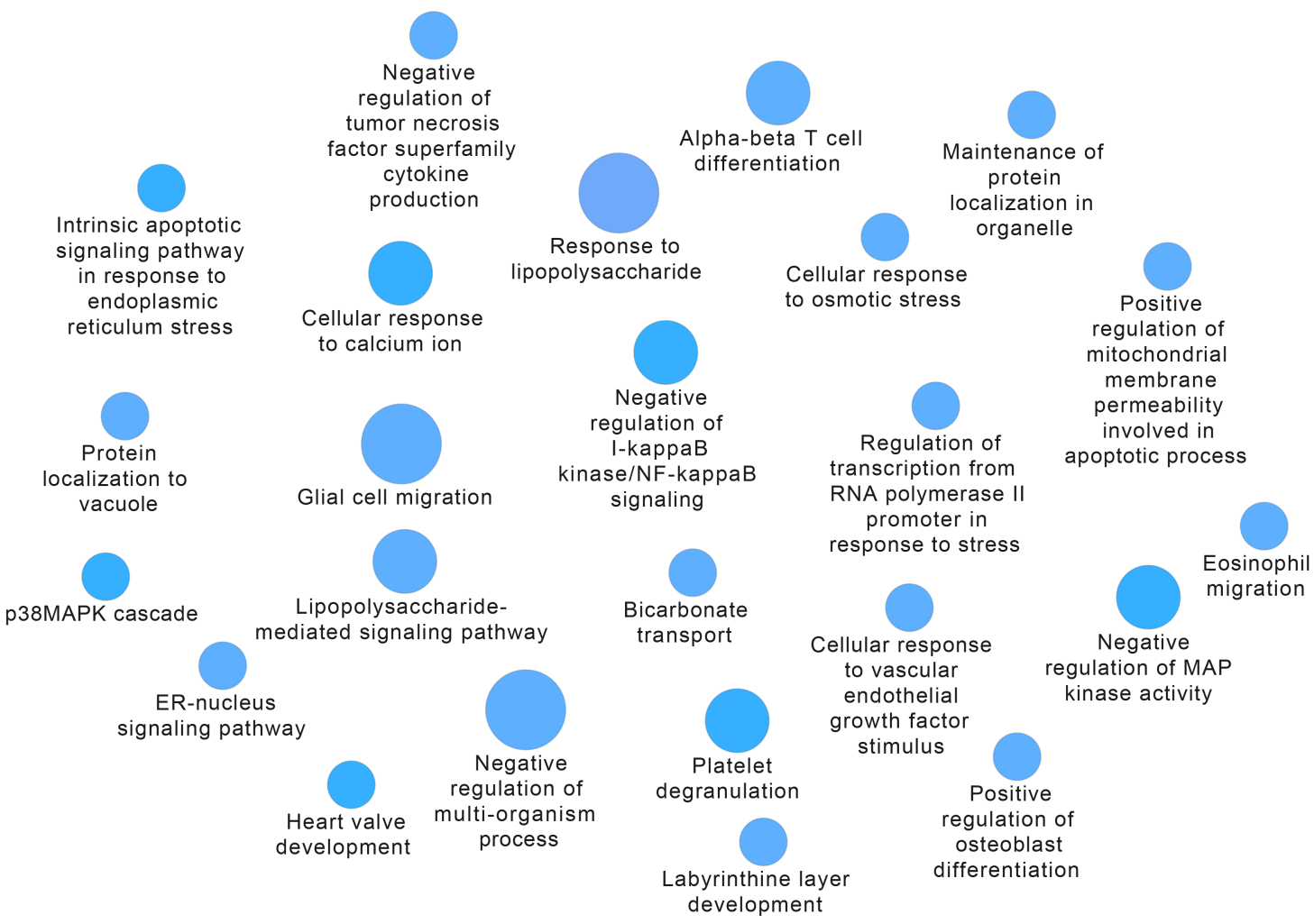

Figure 2. NK cells show a distinct gene expression profile in ARDS patients. (A) NK cells from sepsis-only $(n=4)$ and sepsis+ARDS $(n=3)$ patients are plotted in a t-SNE distribution. (B) The heatmap shows differential gene expression in NK cells in the 2 patient groups. The overall log ${ }_{2}$ FC of combined patient samples is indicated on the right side of the plot. (C) Violin plots of IFNGR1 and IFITM3 in sepsis-only and sepsis+ARDS patients. (D) Significantly downregulated genes in NK cells in ARDS patients were analyzed by ClueGO, with each node representing the identified pathway. The size of the node corresponds to the enrichment significance. 
A

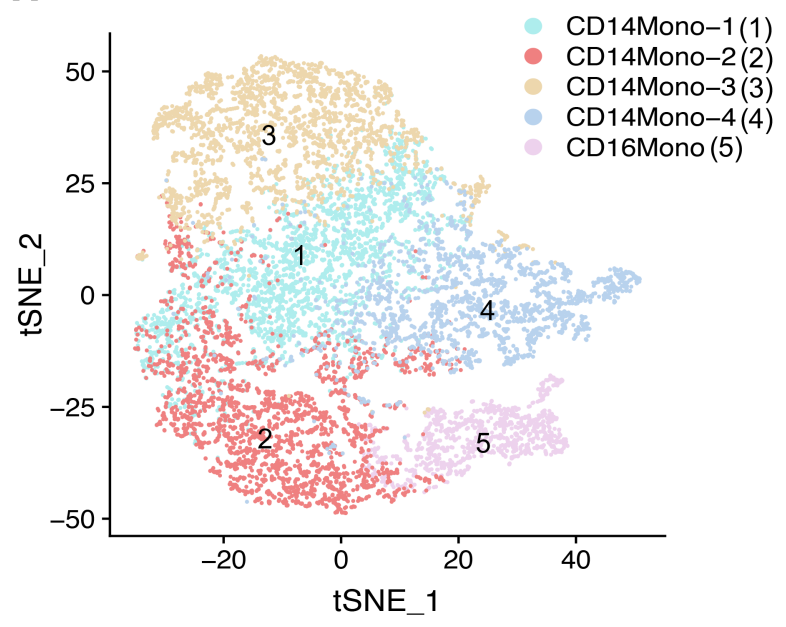

C

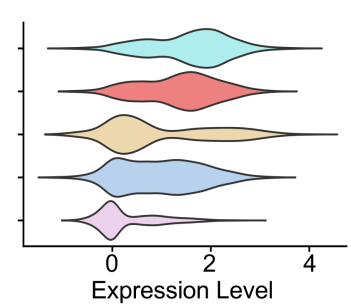

CD74

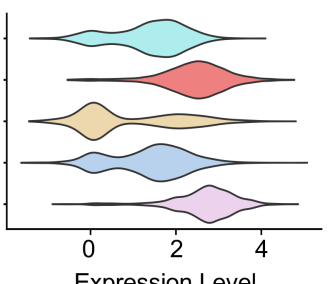

Expression Level
FCGR3A

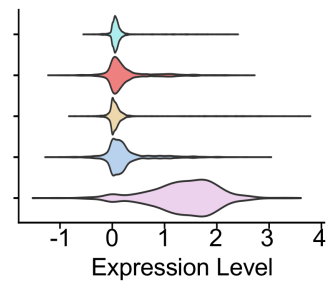

CTSD

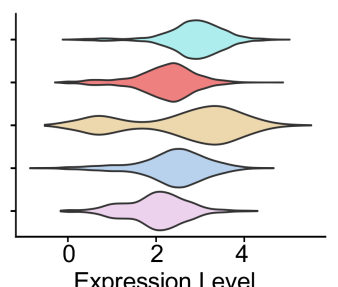

B

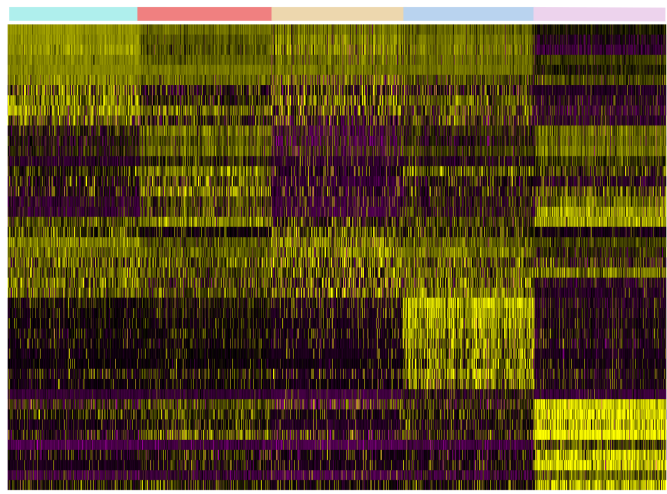

Monocytes ordered by clusters $(n=10367)$

CES1

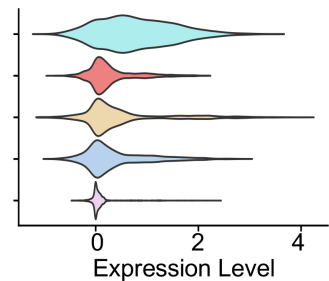

PPBP

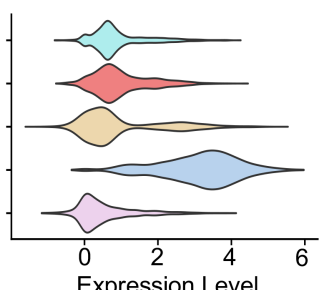

D

E

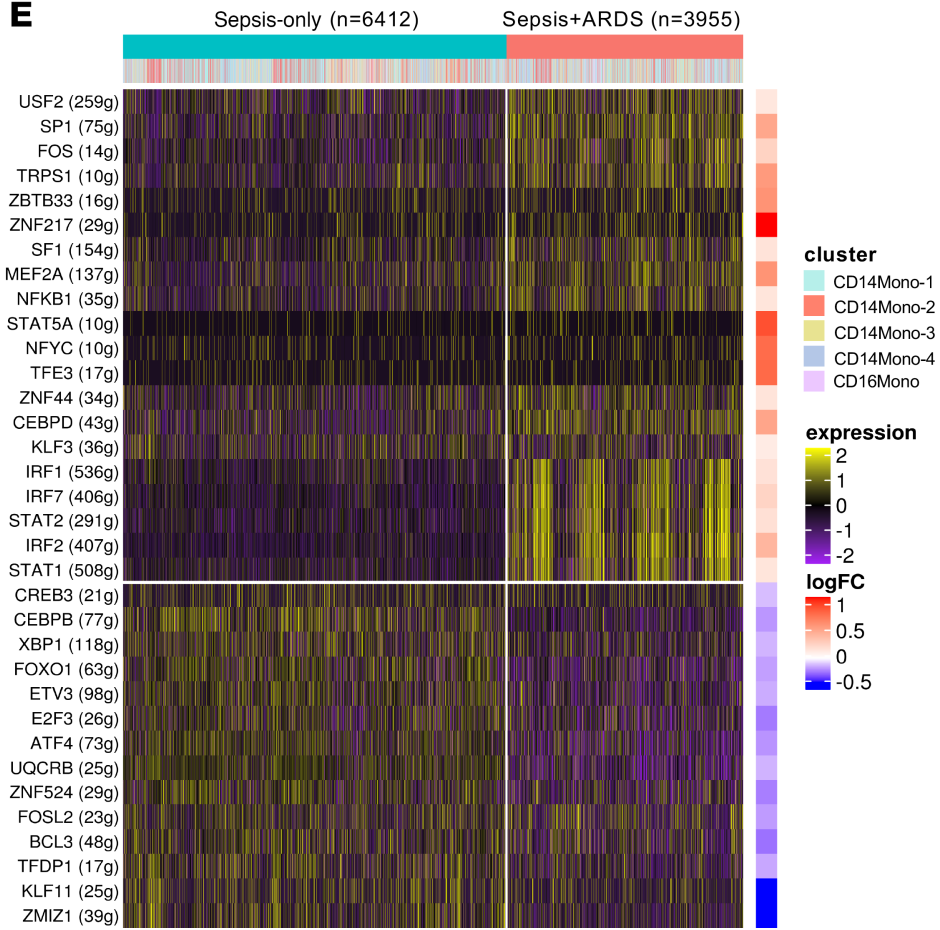


Figure 3. Monocyte clusters are identified in sepsis-only and sepsis+ARDS patients. (A) Monocyte clusters in combined patient samples ( $n=4$, sepsis-only; $n=3$, sepsis+ARDS) are identified by t-SNE distribution. The number in the parenthesis corresponds to the color-coded cell cluster. (B) A heatmap of gene expression in the pooled monocyte clusters is associated with the corresponding cluster in $\mathbf{A}$ using the color-coded bar at the top. (C) Violin plots demonstrate expression of the genes that identify each monocyte cluster. (D) Enriched biologic pathways for upregulated genes in sepsis+ARDS patients are demonstrated using ClueGO biological processes. Each node represents a biological process, and the size of the nodes correspond to the enrichment significance. Colors in the nodes correspond to the monocyte clusters in $\mathbf{A}$. (E) The heatmap demonstrates monocyte transcription factor module expression by patient group and monocyte cluster, with $\log _{2}$ FC illustrated on the right side. Data shown represent results obtained from pooled samples in each group.

showed upregulation of type I IFN signaling pathways. CD74 (cluster 2) and CTSD (cluster 3) monocytes were linked to neutrophil migration and degranulation, respectively. The CTSD (cluster 3) monocyte cluster was enriched in pathways related to host defense, including responses to LPS and virus. Pathways involved in platelet aggregation were upregulated in ARDS in the CD74 (cluster 2) monocyte cluster. Analysis of gene regulatory networks in monocytes revealed transcription factors that are differentially activated in monocytes of ARDS versus sepsis-only patients (Figure $3 \mathrm{E}$ ). The IFN regulatory transcription factor (IRF) family proteins IRF1, IRF2, and IRF7 and STAT family (STAT1, STAT2) were activated in the monocyte populations of ARDS patients, while $C / E B P / \beta, F O X O 1$, and $A T F 4$ were suppressed in these cells. By RNAseq, we previously showed that human alveolar macrophages exhibit a strong type I IFN gene signature and increased IRF7 expression when stimulated by LPS ex vivo (25).

SOCS3 expression is downregulated in the monocytes of ARDS patients. We next examined whether increased expression of IFN-induced genes in the ARDS group was caused by a deficiency of negative regulators of JAK-STAT signaling such as SOCS3. As published previously, SOCS3 efficiently blocks type II IFN-induced (IFN- $\gamma$-induced) responses in cells stimulated by IL-6 (26). Deletion of SOCS3 in macrophages was shown to induce aberrant STAT1 activation in response to IL-6 (27). In subsequent studies, SOCS3 was also shown to inhibit type I IFN-induced (IFN- $\beta$-induced) signaling in macrophages (28). Given that serum IL-6 and IFN- $\gamma$ levels have been associated with poor prognosis in ARDS $(29,30)$, we examined whether the monocyte populations in the ARDS patients had a defect in SOCS3 expression as compared with cells from patients with sepsis only. This analysis showed that indeed SOCS3 expression was barely detectable in all of the monocyte clusters in ARDS patients, while robust expression was detected in cells from patients with sepsis only (Figure 4). Since SOCS1 also inhibits type I (31) and type II (32) IFN signaling, we also examined its expression in monocytes (Supplemental Figure 4A). SOCS1 expression, however, was low in all CD14- and CD16-expressing monocyte clusters. We next examined IFN receptor expression and whether genes that are known to be induced by IFN- $\gamma$ or type I IFNs are differentially expressed in the cells from both groups of patients. While all monocyte clusters were found to express IFNGR 1 at similar levels, expression of IFNAR1 was low (Supplemental Figure 4). Low expression of IFNAR1 may be due to ligand-mediated downregulation of the receptor, as was recently reported for IFNGR1 in human monocytes (24). Expression of GBP1 - which encodes a member of the family of guanylate-binding proteins (GBPs), with functions in host defense $(33,34)$ was expressed at a higher level in $\mathrm{CD}_{1} 6^{+}$monocytes, and the expression of multiple ISGs was also higher in the cells of ARDS patients (Figure 4). Treatment of human monocytes with IFN- $\beta$ was previously shown to upregulate genes such as GBP1, IFITs, and IFI44L (35). Some of these IFN-target genes (GBP1, IFITM1) were found to be preferentially upregulated in $\mathrm{CD}^{+} 6^{+}$monocytes. However, the expression of GABARAP - which is an IFN- $\gamma$-activated gene and shown to be important for cellular distribution of GBPs (36) and antimicrobial responses (36), or of $L Y N$, another IFN- $\gamma$-induced gene (37) — was also similar in the cells from both groups (Figure 4). Taken together, these data identify a molecular defect in the expression of SOCS3 in circulating monocytes of ARDS patients, which our data suggest has downstream consequences that include upregulation of multiple genes, primarily targets of type I IFNs.

Differential monocyte gene expression identifies a predictive score to identify ARDS from bulk transcriptomic data. The data shown above identify a preferential upregulation of type I IFN-regulated genes in the CD16 monocyte cluster, which comprises $\sim 10 \%$ of all monocyte populations in the peripheral circulation of humans. We next asked whether it was possible to identify a core gene signature set that distinguished the transcript profile of the total monocyte population in ARDS patients as compared with that in patients with sepsis only but at risk for ARDS. We identified DEGs in monocytes in our data set as candidates for generating an ARDS risk score (Figure 5A and Supplemental Table 6). The weight of each gene was calculated as $\log$ FC. In our scRNA-seq data, we identified 29 genes that were selectively differentially regulated in the monocytes of ARDS patients compared to sepsis-only patients, with Bonferroni-corrected $P<0.01$ in both 
socs3

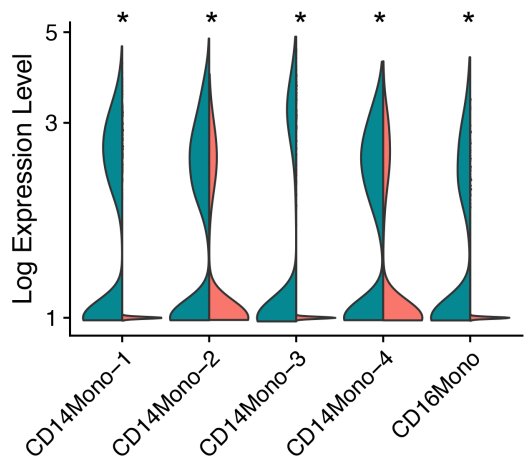

GBP2

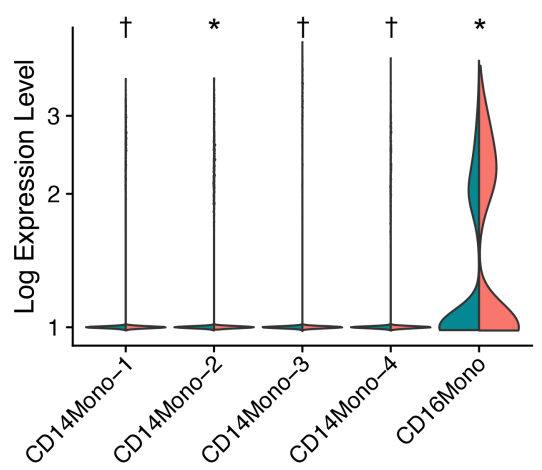

IFITM1

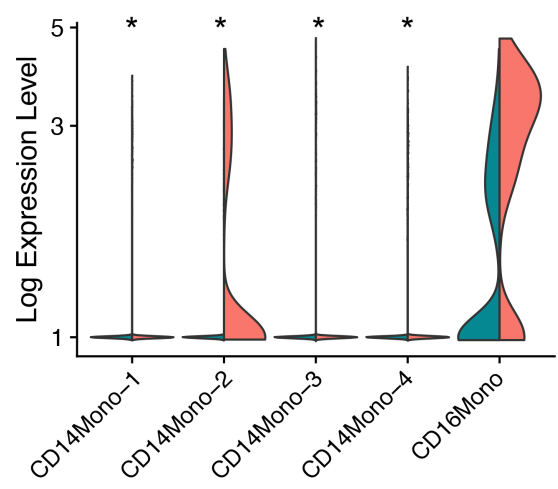

GABARAP

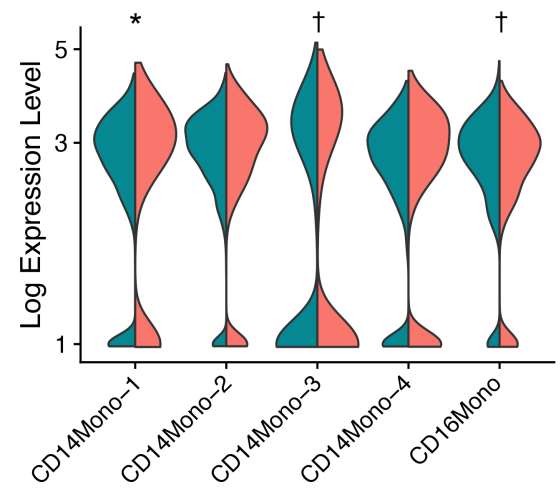

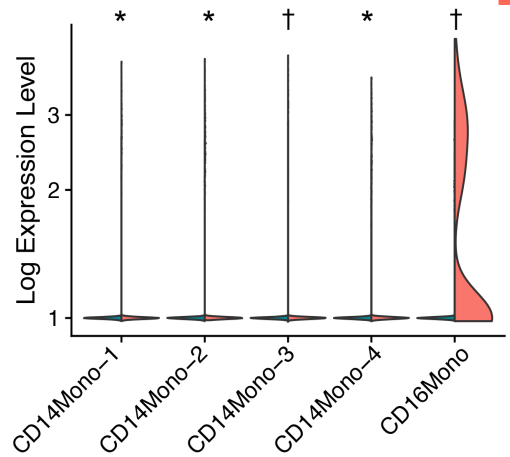

IFI44L

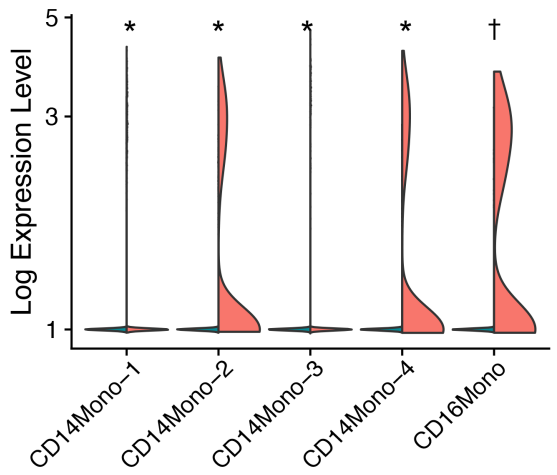

ISG20

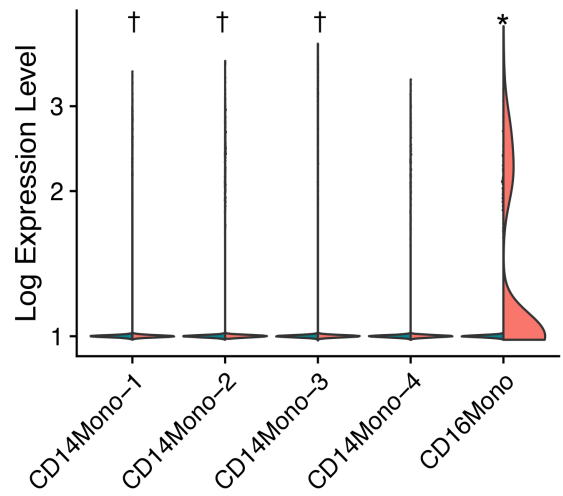

$L Y N$

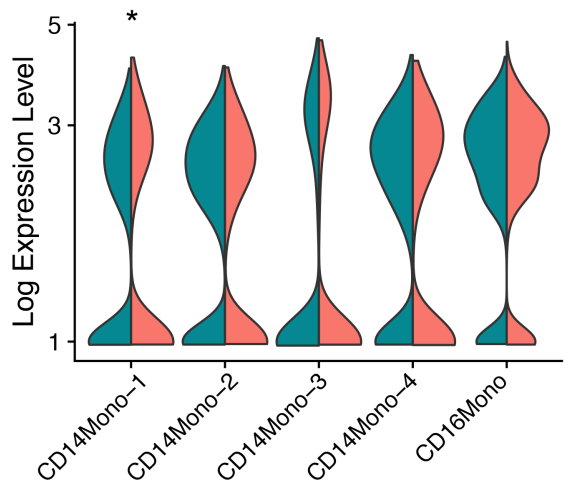

Sepsis-only Sepsis+ARDS
Figure 4. Downregulation of SOCS3 expression in monocyte clusters and increase in expression of

IFN-stimulated genes. Violin plots of expression of selected genes in the monocyte clusters are shown for sepsis-only $(n=4)$ and sepsis+ARDS $(n=3)$ patients. ${ }^{*} P<0.01$, representing genes significantly differentially expressed between sepsis-only and sepsis+ARDS patients within each monocyte population, at Bonferroni-corrected $P$ value in MAST but not in Wilcoxon rank sum test; $\uparrow P$ $<0.01$, representing significance at Bonferroni-corrected $P$ value in both MAST and Wilcoxon rank sum test. 
A

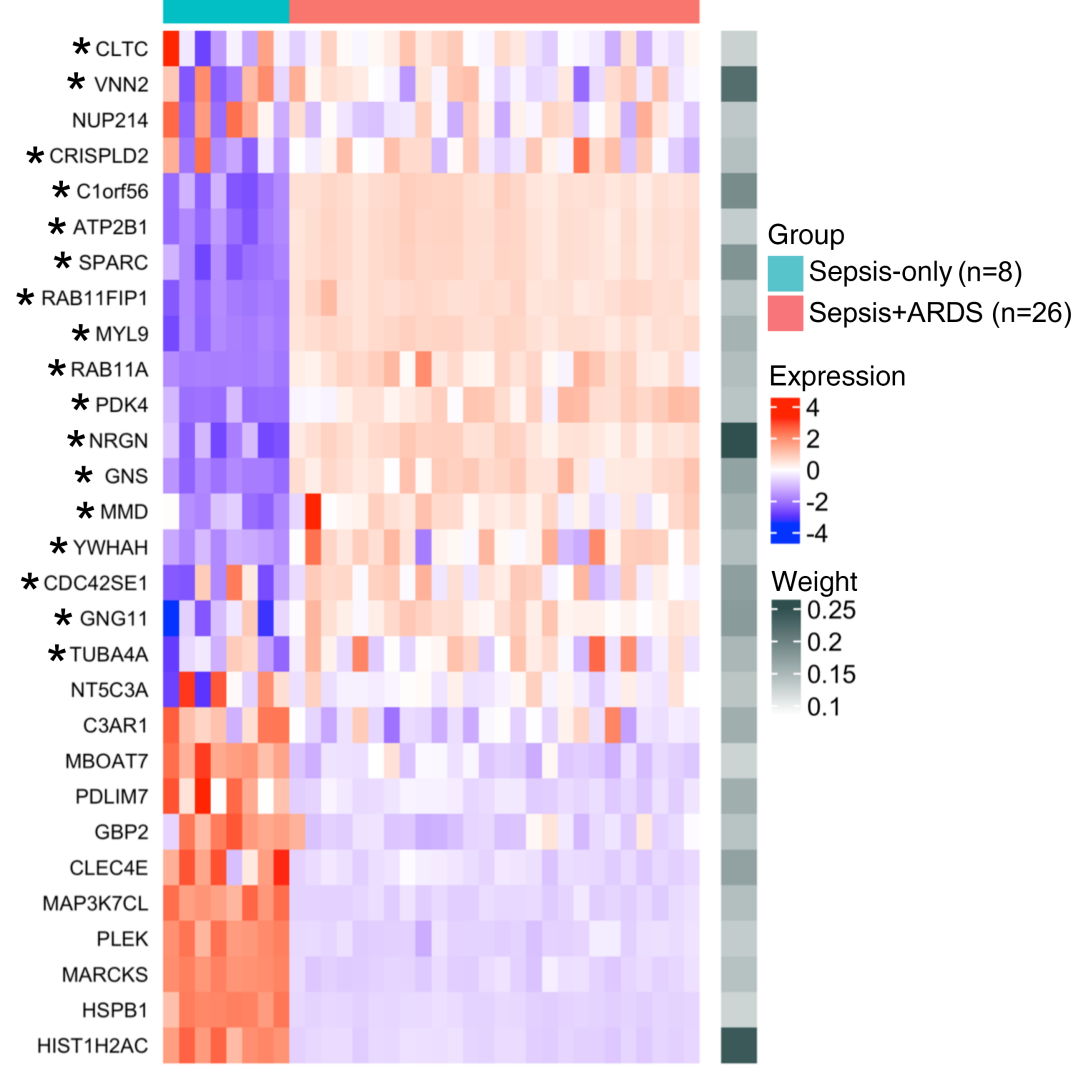

B

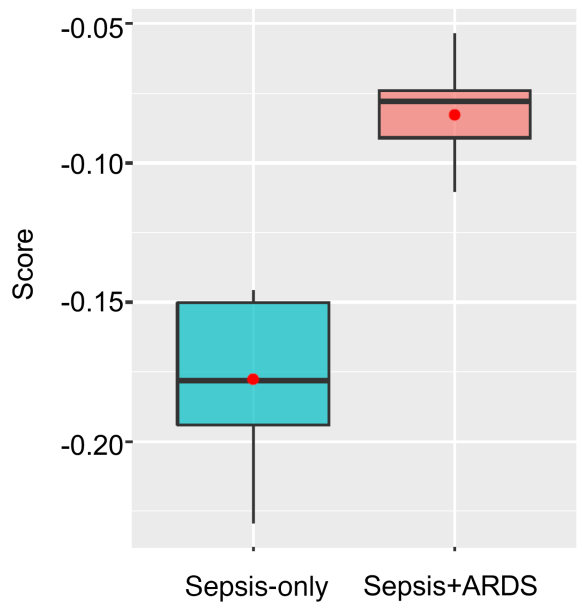

C

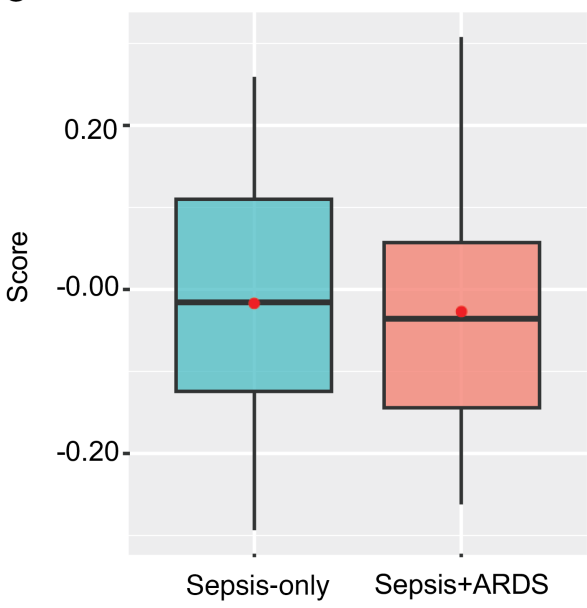

Figure 5. Differential gene expression in monocytes can distinguish between sepsis-only and sepsis+ARDS patients.

(A) Heatmap showing expression levels and weights assigned to each of the 29 genes that distinguish between patients with sepsis only $(n=8)$ and those with sepsis+ARDS $(n=26)$ in our scRNA-seq data as compared with microarray gene expression data of peripheral blood monocytes that were publicly available in 2 data sets. Asterisks indicate genes upregulated in both the scRNA-seq data set and publicly available data sets. (B and C) Comparison between groups using the ARDS risk score comprising 29 genes (B) versus a random selection of genes (C). Box-and-whisker plots show the median (bar) with IQR (box) and upper/lower limit within 1.5 IQRs from the box range (whiskers). The red dot indicates the mean of the score values. In B, values of the box plot for scores of sepsis-only patients are: minimum $=-0.23$, lower $=-0.19$, middle $=$ -0.18 , upper $=-0.15$, maximum $=-0.15$. For sepsis+ARDS patients: minimum $=-0.11$, lower $=-0.09$, middle $=-0.08$, upper $=-0.07$, maximum $=-0.05$. In C, values of the box plot for scores of sepsis-only patients are: minimum $=-0.29$, lower $=-0.12$, middle $=$ -0.01 , upper $=0.11$, maximum $=0.26$. For sepsis+ARDS patients: minimum $=-0.26$, lower $=-0.14$, middle $=-0.04$, upper $=0.06$, maximum $=0.31$. Two-tailed Student's $t$ test was applied.

Wilcoxon rank number test and MAST (38) and with $\operatorname{logFC}$ greater than 0.13 (Figure 5A). One of these genes, $R A B 11 A$, is a small GTPase that inhibits neutrophil efferocytosis, and targeting this gene in macrophages was shown to promote resolution of LPS-induced lung injury (39). Increased expression of $R A B 11 A$ in cells of ARDS patients, therefore, can be expected to result in increased accumulation of neutrophils in the peripheral blood with potential for increased pulmonary infiltration. Related to this possibility, we also identified increased expression of the gene $A T P 2 B 1$, encoding a calcium pump (40), in the monocytes of ARDS patients, the functional correlate of which would be increased cellular export of calcium with the potential to impact neighboring cells, including endothelial cells. Increased calcium signaling in endothelial cells can cause endothelial barrier disruption, which is a feature of ARDS (41). Additional genes of interest that were expressed at a higher level in the cells of ARDS patients included SPARC, which promotes processing of procollagen to collagen (42), with lung fibrosis being a feature of ARDS. Our data suggest that 
both fibroproliferation and monocyte-expressed SPARC may contribute to an increase in fibrosis in ARDS patients. SPARC was recently identified as a marker of myeloid-derived suppressor cells (MDSCs) (43), and interestingly, we detected increased expression of another gene, $N R G N$, that has been also found to be expressed by MDSCs (Gabrilovich Condamine US patent 2018). MDSC expansion has been associated with nosocomial infections in patients with sepsis (44). Increased expression of PDK4 in cells of the ARDS group may contribute to increased lactate levels in these patients, which has been associated with increased risk for mortality (45).

We next assessed the relative expression of these 29 genes in peripheral blood monocytes of sepsis-only versus sepsis+ARDS patients using available data sets in the Gene Expression Omnibus (GEO) database (https://www.ncbi.nlm.nih.gov/geo/query/acc.cgi?acc=GSE89953; https://www.ncbi.nlm. nih.gov/geo/query/acc.cgi?acc=GSE46955). The relevant available human data sets are summarized in Supplemental Table 7. After combining these data sets, 8 sepsis patients with infection by Gram-negative bacteria and 26 ARDS patients were included in the initial analysis. The data sets underwent individual-wise normalization and standardization across genes. Among the 15,555 genes whose expression was available in both data sets, 17 upregulated genes among the 29 differentially expressed in the monocytes of ARDS patients in our data were also upregulated in the cells of ARDS patients in the bulk RNA expression data sets (Figure 5A and Supplemental Table 6). A significant difference was observed between scores of the septic group versus the ARDS group $\left(P<2.2 \times 10^{-16}\right.$, Figure 5B). No significant difference in expression was evident between scores when genes were randomly selected from the groups in a sensitivity analysis $(P=0.600$, Figure $5 \mathrm{C})$. Of note, due to the high stringency set for this analysis with the application of both Wilcoxon rank sum test and MAST, and also a cutoff of fold change to 0.1 , SOCS3 and IFN-stimulated genes identified as being differentially expressed in the monocytes between the 2 groups using MAST alone (Figure 4), a method tailored for analysis of scRNA-seq data, were precluded in this 29-gene set. The schematic in Figure 6 depicts potential mechanisms by which the DEGs identified in this analysis might impact ARDS.

\section{Discussion}

ARDS is a lethal clinical syndrome that occurs commonly in the ICU, although the pathogenesis in humans is poorly understood. We hypothesized that utilization of single cell transcriptomics early in the disease course of ARDS would lead to the identification of novel molecular signatures that characterize ARDS. To the best of our knowledge, our study is the first to apply scRNA-seq technology to a cohort of patients with pneumonia and sepsis who have advanced to ARDS and those with pneumonia and sepsis only but who are at risk for ARDS and identify distinct molecular profiles in their peripheral blood immune cells. In a prior study of this cohort comparing patients with a known risk factor for ARDS but not meeting clinical Berlin criteria for the diagnosis of ARDS (18) to patients who developed ARDS, the 2 groups were indistinguishable by clinical and biomarker parameters (46). The present study shows that ARDS and patients at risk for ARDS can be distinguished at the cellular and molecular levels using the finer resolution afforded by scRNA-seq technology. The results of our analysis could form the basis for biomarker discovery aimed at distinguishing ARDS from respiratory complications without ARDS.

Our data advance prior bulk RNA-seq analyses comparing sepsis-only with sepsis+ARDS by being able to distinguish between cellular composition and gene expression in cells from the 2 groups. At the cellular abundance level, we have identified a reduced frequency of peripheral blood NK cells in ARDS patients as compared with that in the other group. However, despite reduced frequency, the NK cells in the ARDS group appeared to have a higher activation profile based on marked downregulation of IFNGRI expression and also of multiple negative regulatory pathways that determine activation of transcription factors such as NF- $\mathrm{kB}$ and of MAP kinases that promote inflammation. In our analysis, increased expression of IFITM3 in NK cells of ARDS patients, which is primarily induced by type I IFNs as an antiviral factor (47) and which inhibits cell proliferation (48), may explain the reduced frequency of the cells in this group. Also, downregulation of IFNGR1 suggests an inability to regulate inflammatory pathways induced by IFN- $\gamma$, as recently demonstrated for human monocytes (24).

One distinct feature of cells from ARDS patients that we have identified is a heightened response to IFNs. The overall analysis of DEGs in our study suggests differential upregulation of type I IFN-induced pathways that include expression of transcripts for GBP family members ISGs and HLA. Increased expression of $H L A-D Q b 1$ was previously described in an ARDS cohort, suggesting increased functional activation 


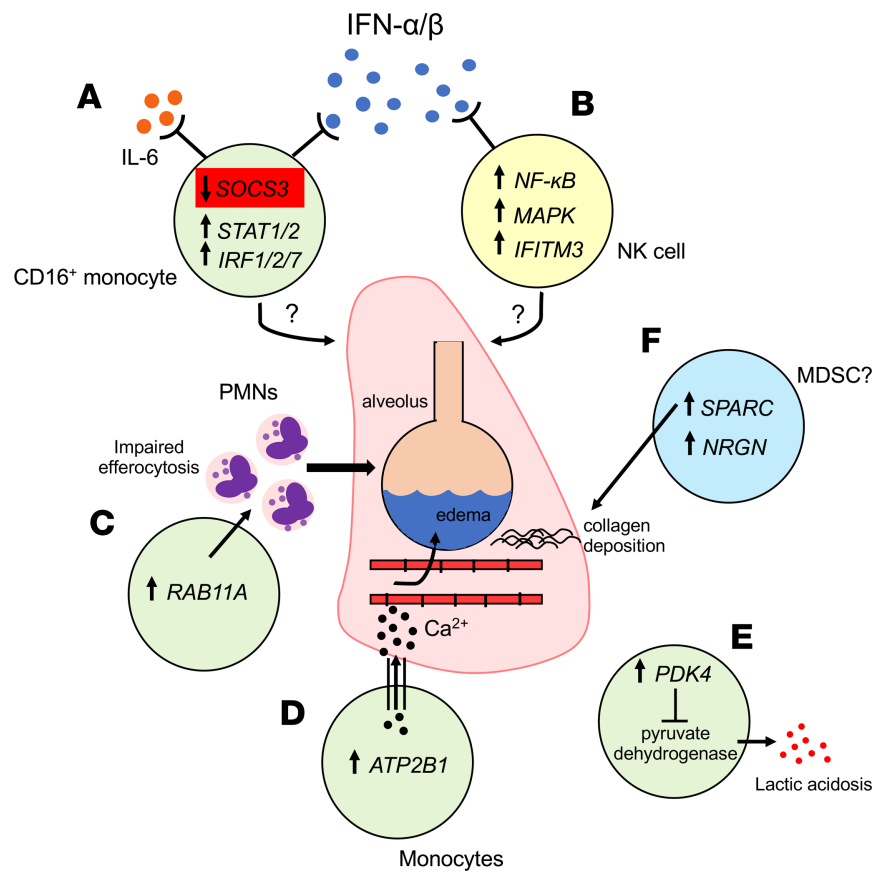

Figure 6. Potential immune mechanisms contributing to the development of ARDS. (A) Monocyte subsets (particularly CD16 monocytes) downregulate SOCS3 expression, which promotes type I IFN signaling through STAT and IRF pathways. Reduced SOCS3 levels may also modulate IL-6 signaling. (B) NK cells have increased expression of canonical activation signaling cascades including NF- $\mathrm{KB}, \mathrm{MAPK}$, and IFN-stimulated genes (IFITM3). (C) Monocytes with increased expression of RAB11A may impair neutrophil efferocytosis, leading to persistent alveolar inflammation. (D) Increased ATP2B1 is involved in calcium efflux, which could contribute to alveolar capillary leak and the development of noncardiogenic pulmonary edema. (E) Increased PDK4 could contribute to lactic acidosis through inhibition of pyruvate dehydrogenase. (F) Monocytes with increased SPARC and NRGN expression could be markers of myeloid-derived suppressor cells (MDSCs). SPARC is involved in collagen deposition, which is observed in the fibroproliferative stage of ARDS.

of monocytes as antigen-presenting cells $(49,50)$. Circulating monocytes can also differentiate into DCs when recruited to the lung (50). Whole blood microarray studies have previously identified neutrophil-related genes to be differentially regulated in sepsis-associated ARDS (16), and pathway analysis identified viral infection to be notably upregulated in ARDS. Furthermore, neutrophil ISG over- and underexpression is associated with reduced survival in ARDS (51). Our data demonstrating activation of type I IFNinduced pathways support the importance of exaggerated type I IFN response in the development and outcome of ARDS. Of note, tonic type I IFN is known to be important for immune homeostasis (52), but unregulated signaling downstream may be detrimental, as suggested by our data. Along these lines, we show deficient SOCS3 expression in the peripheral blood monocytes of ARDS patients. SOCS3 negatively regulates signaling downstream of IL- 6 and also type I and type II IFNs. Interestingly, SOCS3 deficiency causes IL- 6 to induce an IFN- $\gamma$-like response in monocytes via STAT1 activation (26). Our findings are further supported by the finding that SOCS3 deficiency in Ly6 $\mathrm{C}^{+}$myeloid cells enhances experimental LPS-induced acute lung injury (53). Given that the genes GBP1, IFI44L, IFITM1, and ISG20 - found to be differentially upregulated in the monocytes of ARDS patients, particularly in the CD16 subset are known to be type I IFN inducible (35), it appears that SOCS3 deficiency in the monocytes of ARDS patients renders them more responsive to circulating levels of type I IFNs than to increased IL-6-mediated IFN- $\gamma$-like signaling. Similar expression of GBP2 (54), GABARAP(36), and LYN(55) in the monocytes of the 2 groups of subjects, genes typically associated with IFN- $\gamma$ signaling, suggests absence of enhanced response to IFN- $\gamma$ in the monocytes of ARDS patients. SOCS3 expression has been inversely associated with the expression of multiple miRNAs in different cell types (56-58). In addition, epigenetic modification of the SOCS3 gene involving promoter hypermethylation was also associated with suppression of SOCS3 expression, especially in cancer $(59,60)$. It would be interesting to determine in future studies the molecular mechanisms that underlie low SOCS3 expression in the monocytes of ARDS patients. Taken together, we propose a SOCS $3^{10}$ signature in peripheral blood monocytes as a biomarker of ARDS.

We identified 4 clusters of $\mathrm{CD} 14^{+}$monocytes and 1 cluster of $\mathrm{CD} 16^{+}$monocytes in our patient cohort. The frequency of each monocyte cluster was not significantly different in ARDS. A third intermediate $\mathrm{CD} 14^{+} \mathrm{CD} 16^{+}$monocyte subset that has been described in the peripheral blood of humans was not detected in our analysis. Monocytes are crucial regulators of immunity (61). Peripheral blood monocytes have been validated to play an important role in LPS-induced lung injury in experimental models. Along with resident alveolar macrophages and recruited alveolar monocytes, they may direct neutrophil-mediated lung injury that characterizes ARDS development $(62,63)$. A prior study associated recruited monocytes/immature macrophages in the BAL fluid of sepsis patients with ARDS with 
decreased survival (9). As presented in our data, increased expression of specific genes in peripheral blood monocytes of ARDS patients may play an important role in multiple aspects of ARDS pathogenesis, including endothelial cell leak and increased neutrophil recruitment to the lungs. In addition to identifying a heightened type I IFN response, preferentially in the $\mathrm{CD}_{16} 6^{+}$subset, we detected upregulation of platelet aggregation pathways in the $\mathrm{CD} 74^{\mathrm{hi}} \mathrm{CD} 14^{+}$monocyte cluster 2 . Platelets are believed to play a role in ARDS pathogenesis through their interaction with the immune system (64).

Monocytes from sepsis+ARDS patients as a whole displayed differential expression of 29 genes as compared with monocytes from the sepsis-only group. This differential expression profile may have important functional ramifications in ARDS (Figure 6). One such gene upregulated in the cells of the ARDS group is PDK4, which inhibits pyruvate dehydrogenase (65), thereby limiting entry of pyruvate into the TCA cycle and causing increased production of lactate. Increased PDK 4 expression in the monocytes of ARDS patients may underlie increased pulmonary and blood lactate levels in ARDS patients $(45,66)$. We also detected higher expression of $A T P 2 B 1$ in the monocytes of ARDS patients. ATP2B1 is a calcium transporter that was shown to couple ATP hydrolysis with the transport of calcium from the cytoplasm to the extracellular space in endothelial cells, thereby maintaining intracellular calcium homeostasis (40). Increased $\mathrm{Ca}^{2+}$ signaling has been implicated in proinflammatory responses and barrier disruption in endothelial cells in ARDS (41), in which monocyte-expressed ATP2B1 may play an important role. Our data also show increased expression of $R A B 11 A$ that encodes a central recycling endosome component (67) in the monocytes from ARDS patients. The same was true for RAB11FIP1 (RAB family interacting protein 1), a key effector molecule for RAB11A. RAB11A promotes cycling of the metalloproteinase ADAM17 from the cytoplasm to the cell surface, which causes proteolytic cleavage of CD36 from the cell surface. CD36 facilitates phagocytosis of apoptotic neutrophils, and it has been shown that RAB11A tonically inhibits macrophage phagocytic functions important for removal of apoptotic neutrophils (39). Interestingly, 17 of these genes in the 29-gene set differentially expressed in the monocytes of ARDS patients in our data set were also found to be expressed at a higher level in the PBMCs of ARDS patients in independent patient cohorts. Collectively, the gene expression profile shows increased monocyte expression of RAB11A implicated in defective clearance of neutrophils and an increase in ATP2B1 linked to vascular leak in ARDS.

Although these data identify potentially novel mechanisms that underlie ARDS pathogenesis, there are limitations. While the small sample size increases the opportunity for influence of confounding factors due to the clinical characteristics of the patients and treatments that cannot be adequately controlled, prior studies using this technology have drawn biologically relevant conclusions from similar patient numbers $(68,69)$. In addition, we validated DEGs in our study across publicly available bulk RNA data sets generated from microarray analysis of RNA isolated from monocytes of patients with sepsis only and with sepsis+ARDS. Despite these limitations, the striking ability to differentiate between sepsis and ARDS using a monocyte gene signature derived from our single cell data highlights the importance of studying peripheral blood monocytes in ARDS, which could be useful for the generation of a diagnostic biomarker panel for individuals at risk for developing ARDS. While caution must be used when comparing transcriptomic profiles of circulating monocytes and alveolar macrophages to extrapolate mechanisms of pathogenesis (70), results of a prior study have suggested the role of recruited monocytes in poor survival of ARDS patients (9). Also, our ability to clearly differentiate between sepsis and ARDS using peripheral blood samples has strengths in ease of sample accessibility. Future studies assessing single cell transcriptomics of alveolar cell populations are clearly warranted, given prior demonstration of the prognostic utility of studying alveolar myeloid populations $(71,72)$.

Overall, our findings achieve a cell-specific resolution of transcriptomics not previously attempted in ARDS that enrich the mechanistic understanding of genes associated with ARDS risk and mortality (73). We identify increased type I IFN signaling in ARDS that may be caused by downregulation of SOCS3 expression in the cells of ARDS patients that triggers a proinflammatory phenotype facilitated by increased activation of IRF and STAT transcription factors. We highlight a SOCS3 ${ }^{\text {lo }}$ phenotype of monocytes as a potential biomarker of ARDS. In addition, we show elevated expression of multiple genes in the monocytes of ARDS patients that, together, may play important roles in precipitating ARDS. In addition, we show reduced frequency of NK cells and complete lack of IFNGR1 expression as additional features of ARDS. These data add to observations in prior studies identifying a single nucleotide polymorphism in MAP kinase kinase kinase 1 (MAP3K) correlated to ventilator-free days in ARDS (74) and 
plasma angiopoietin-2 as a potential causal biomarker of sepsis-induced ARDS (75). While only patients with pneumonia as an ARDS risk factor were studied, these findings have important implications in the pathogenesis of ARDS overall, as well as in identifying potential pathways to therapeutically modify disease course and reduce the burden of ARDS in the ICU. Future temporal studies of patient samples by single cell transcriptomics that would include patients with various ARDS risk factors would help to more completely characterize the immunopathology of ARDS.

\section{Methods}

All reagents and materials used in this study are listed in Supplemental Table 8.

Study design and sample collection. Patient samples were selected from critically ill mechanically ventilated patients aged > 18 years old in the University of Pittsburgh Medical Intensive Care Unit that were prospectively enrolled in the Acute Lung Injury Registry as described previously (46). Blood samples were obtained at the time of enrollment, and patients were assigned to either at risk for ARDS with sepsis (sepsis only) and 3 who had ARDS with sepsis (sepsis+ARDS) meeting the Berlin criteria (18) based on a retrospective review by a consensus committee of critical care physicians. Patients at risk for ARDS with sepsis caused by pneumonia were selected in an attempt to reduce confounding signals from other sources of sepsis. Sepsis was defined as organ dysfunction manifested by the need for mechanical ventilation in the setting of a dysregulated immune response to presumed or proven infection (pneumonia) (76). Blood samples collected $>24$ hours from the time of intubation were excluded. Patients were excluded if they were recipients of a transplanted organ, had active malignancy, or received systemic immunosuppression or systemic glucocorticoids.

PBMC isolation and cryopreservation. Peripheral blood was drawn into a tube with acid citrate dextrose (ACD) and then transferred to a $50 \mathrm{~mL}$ Falcon tube. Blood was diluted approximately 1:1 with room temperature sterile PBS-LPS tested. After layering Ficoll Paque PLUS, the samples were centrifuged at $450 \mathrm{~g}$ for 30 minutes at $25^{\circ} \mathrm{C}$ in a swinging-bucket rotor without braking. The mononuclear cell layer at the interphase was transferred and washed 3 times with $15 \mathrm{~mL}$ PBS. PBMC were resuspended in cold freeze medium containing $10 \%$ dimethyl sulfoxide, 40\% FBS, and 50\% X-VIVO15 medium and slowly cooled to $-80^{\circ} \mathrm{C}$ in a Nalgene Cryo $1^{\circ} \mathrm{C}$ freezing container. Samples were stored in liquid nitrogen. PBMCs were thawed slowly in a $37^{\circ} \mathrm{C}$ water bath and transferred drop-wise to warm complete RPMI containing $10 \%$ human $\mathrm{AB}$ serum and 0.05M 2-mercaptoethanol. PMBCs were spun down at $350 \mathrm{~g}$ for 7 minutes at $4^{\circ} \mathrm{C}$ and gently resuspended in PBS with $0.04 \%$ BSA. As previously reported (77), our pilot studies comparing fresh and cryopreserved cells from healthy controls closely matched each other in average gene expression; therefore, only cryopreserved cells of all patients were used for scRNA-seq in this study.

scRNA-seq and quality control. scRNA-seq was performed using 10× Genomics Single Cell 3' solution, version 2, according to the manufacturer's instructions, with 7000 cells loaded for each sample $(n=4$ sepsis-only and $n=3$ sepsis + ARDS). Libraries were sequenced on the HiSeq Platform (Illumina) from Novogene and raw BCL/fastq files were analyzed using Cell Ranger version 3.0.2 (10× Genomics). Low-quality cells were removed if they expressed $<3000$ genes/cell and had more than $20 \%$ mitochondrial and 10\% hemoglobin gene expression. After quality control, 24,806 cells with mean reads 50,419/ cell remained for downstream bioinformatic analyses.

Data processing and bioinformatic analysis. Samples were analyzed using Seurat (version 3.0, https://satijalab.org/seurat/) for batch correction and cell type identification (78). Alignment between samples was achieved by identifying gene-to-gene correlation patterns that are conserved across data sets through canonical correlation analysis (CCA). The cells were then embedded in a shared low-dimensional space and normalized using nonlinear "warping" algorithms (78). We subsequently identified discrete cell populations by a shared-nearest neighbor (SNN) graph-based clustering method. Differential gene expression analysis was applied in each cell type, with 2 methods as reciprocal validation: Wilcoxon rank number test and MAST (38), the former as the most commonly used and the latter customized for scRNA-seq data. Pathway enrichment analysis was further conducted with ClueGO (79) based on GO biological process databases (04/09/2018).

Functional analysis of subpopulations in monocytes. Differential gene expression analysis was performed in each of the monocyte clusters, and the log-transformed fold changes $\left(\log _{2} \mathrm{FC}\right)$ were used to perform hierarchical clustering between genes. Five sets of genes were identified and demonstrated different expression patterns between monocytes from sepsis-only and sepsis+ARDS patients. Top DEGs in each subcluster tested for enrichment of biological pathways with ClueGO. Gene regulatory networks were analyzed by SCENIC (80) within monocytes. 
Validation in GEO bulk RNA data sets. An ARDS risk score was generated based on the findings from our scRNA-seq data. Significant DEGs in monocytes were selected with Bonferroni-corrected $P$ values less than 0.01 in both Wilcoxon rank number test and MAST and an absolute value of $\log _{2} \mathrm{FC}$ greater than 0.1 . The weight for each gene was calculated as $\log F C$ divided by negative $\log _{10}$ Bonferroni-corrected $P$ value in MAST. The available human monocyte data sets on GEO (GSE89953, GSE46955) were normalized to distribution of zero-mean and $1 \mathrm{SD}$ in each individual. Risk score was calculated as $\mathrm{W} \times \mathrm{E}$, where $\mathrm{W}$ represents the weight of each gene and $\mathrm{E}$ represents the normalized expression matrix. Risk scores are shown in the box plots, stratified by disease status. In addition, sensitivity analysis was performed, with scores generated using randomly selected gene sets of the same number with the same weights for 10 replicates to reduce the potential bias in the data sets.

Data availability. Raw scRNA-seq data (fastq files) and processed UMI matrices from our scRNA-seq experiment are available on GEO (GSE151263). Two public human RNA microarray data sets can be accessed from GEO (GSE89953, GSE46955).

Statistics. Differential gene expression analysis was conducted between identified clusters to find marker genes for each cell type using Wilcoxon rank sum test. Further differential analysis between sepsis-only and sepsis+ARDS patients was applied within each cell type using 2 methods as reciprocal validation: Wilcoxon rank number test and MAST. Bonferroni correction was used to adjust for multiple testing, and the cutoff $P$ value of 0.01 after adjustment was set for all differential expression analysis. Pathway enrichment analysis was conducted with ClueGO (79), which uses the Fisher exact test with cutoff as Bonferroni-corrected $P<0.01$. GEO data set gene expression significance in Supplemental Table 6 was performed using a Student's $t$ test with 2 tails.

Study approval. Informed consent was obtained from all patients or surrogates enrolled in the Acute Lung Injury Registry. The study was approved by the IRB of the University of Pittsburgh (IRB no. 19050099).

\section{Author contributions}

WC, AR, and PR conceived and designed the study. YJ, BRR, JC, and SD conducted the primary analysis and interpreted data. JSL, GDK, RKM, and BJM participated in data analysis. YJ and BRR prepared the first draft of the manuscript. All authors reviewed the draft for intellectual content and approved submission of the final version of the manuscript.

\section{Acknowledgments}

This study was supported by grants HLP01114453 (PR, BJM, JSL), F32HL152497 and LRP HLHEPR8727 (BRR), and 2T32HL007563-31 (BRR) from the U.S. NIH. YJ was supported by the Tsinghua Educational Foundation of North America (TEFNA) during her training at the University of Pittsburgh through the Pitt-Tsinghua collaborative program. The authors thank Sarah Rapport for management of the Acute Lung Injury Registry and assistance with patient consent.

Address correspondence to: Prabir Ray, Division of Pulmonary, Allergy and Critical Care Medicine, University of Pittsburgh, NW 628 UPMC Montefiore, 3459 Fifth Avenue, Pittsburgh, Pennsylvania 15213, USA. Phone: 412.802.3192; Email: rayp@pitt.edu. Or to: Wei Chen, Division of Pulmonary Medicine, UPMC Children's Hospital of Pittsburgh, Room 9125, Rangos Research Building, 4401 Penn Avenue, Pittsburgh, Pennsylvania 15224, USA. Phone: 412.692.6241; Email: wei.chen@chp.edu.

\footnotetext{
1. Bellani G, et al. Epidemiology, Patterns of Care, and Mortality for Patients With Acute Respiratory Distress Syndrome in Intensive Care Units in 50 Countries. JAMA. 2016;315(8):788-800.

2. Fan E, Brodie D, Slutsky AS. Acute Respiratory Distress Syndrome: Advances in Diagnosis and Treatment. JAMA 2018;319(7):698-710.

3. Acute Respiratory Distress Syndrome Network, et al. Ventilation with lower tidal volumes as compared with traditional tidal volumes for acute lung injury and the acute respiratory distress syndrome. N Engl J Med. 2000;342(18):1301-1308.

4. National Heart, Lung, Blood Institute Acute Respiratory Distress Syndrome (ARDS) Clinical Trials Network, et al. Comparison of two fluid-management strategies in acute lung injury. $N$ Engl J Med. 2006;354(24):2564-2575.

5. Guérin C, et al. Prone positioning in severe acute respiratory distress syndrome. N Engl J Med. 2013;368(23):2159-2168

6. Matthay MA, et al. Acute respiratory distress syndrome. Nat Rev Dis Primers. 2019;5(1):18.

7. Ware LB, Matthay MA. The acute respiratory distress syndrome. N Engl J Med. 2000;342(18):1334-1349.

8. Thompson BT, Chambers RC, Liu KD. Acute Respiratory Distress Syndrome. N Engl J Med. 2017;377(6):562-572.

9. Rosseau S, et al. Phenotypic characterization of alveolar monocyte recruitment in acute respiratory distress syndrome.
} 
Am J Physiol Lung Cell Mol Physiol. 2000;279(1):L25-L35.

10. Aggarwal NR, King LS, D'Alessio FR. Diverse macrophage populations mediate acute lung inflammation and resolution. Am J Physiol Lung Cell Mol Physiol. 2014;306(8):L709-L725.

11. Zapol WM, Trelstad RL, Coffey JW, Tsai I, Salvador RA. Pulmonary fibrosis in severe acute respiratory failure. Am Rev Respir Dis. 1979;119(4):547-554.

12. Anderson WR, Thielen K. Correlative study of adult respiratory distress syndrome by light, scanning, and transmission electron microscopy. Ultrastruct Pathol. 1992;16(6):615-628.

13. Howrylak JA, et al. Discovery of the gene signature for acute lung injury in patients with sepsis. Physiol Genomics. 2009;37(2):133-139.

14. Dolinay T, et al. Inflammasome-regulated cytokines are critical mediators of acute lung injury. Am J Respir Crit Care Med. 2012;185(11):1225-1234.

15. Rogers AJ, et al. Association of Elevated Plasma Interleukin-18 Level With Increased Mortality in a Clinical Trial of Statin Treatment for Acute Respiratory Distress Syndrome. Crit Care Med. 2019;47(8):1089-1096.

16. Kangelaris KN, et al. Increased expression of neutrophil-related genes in patients with early sepsis-induced ARDS. Am J Physiol Lung Cell Mol Physiol. 2015;308(11):L1102-L1113.

17. Kan M, Shumyatcher M, Himes BE. Using omics approaches to understand pulmonary diseases. Respir Res. 2017;18(1):149.

18. ARDS Definition Task Force, et al. Acute respiratory distress syndrome: the Berlin Definition. JAMA. 2012;307(23):2526-2533.

19. Englert JA, et al. Whole blood RNA sequencing reveals a unique transcriptomic profile in patients with ARDS following hematopoietic stem cell transplantation. Respir Res. 2019;20(1):15.

20. Garten A, Schuster S, Penke M, Gorski T, de Giorgis T, Kiess W. Physiological and pathophysiological roles of NAMPT and NAD metabolism. Nat Rev Endocrinol. 2015;11(9):535-546

21. Ogata-Suetsugu S, et al. Amphiregulin suppresses epithelial cell apoptosis in lipopolysaccharide-induced lung injury in mice. Biochem Biophys Res Commun. 2017;484(2):422-428.

22. Rajakariar R, et al. Hematopoietic prostaglandin D2 synthase controls the onset and resolution of acute inflammation through PGD2 and 15-deoxyDelta12 14 PGJ2. Proc Natl Acad Sci USA. 2007;104(52):20979-20984.

23. Chen Y, Perussia B, Campbell KS. Prostaglandin D2 suppresses human NK cell function via signaling through D prostanoid receptor. J Immunol. 2007;179(5):2766-2773.

24. Crisler WJ, Eshleman EM, Lenz LL. Ligand-induced IFNGR1 down-regulation calibrates myeloid cell IFN $\gamma$ responsiveness. Life Sci Alliance. 2019;2(5):e201900447.

25. Pinilla-Vera M, et al. Full Spectrum of LPS Activation in Alveolar Macrophages of Healthy Volunteers by Whole Transcriptomic Profiling. PLoS One. 2016;11(7):e0159329.

26. Croker BA, et al. SOCS3 negatively regulates IL-6 signaling in vivo. Nat Immunol. 2003;4(6):540-545.

27. Qin H, Holdbrooks AT, Liu Y, Reynolds SL, Yanagisawa LL, Benveniste EN. SOCS3 deficiency promotes M1 macrophage polarization and inflammation. J Immunol. 2012;189(7):3439-3448.

28. Akhtar LN, et al. Suppressor of cytokine signaling 3 inhibits antiviral IFN-beta signaling to enhance HIV-1 replication in macrophages. J Immunol. 2010;185(4):2393-2404.

29. Shankar-Hari M, Fan E, Ferguson ND. Acute respiratory distress syndrome (ARDS) phenotyping. Intensive Care Med. 2019;45(4):516-519.

30. Bos LD, et al. Identification and validation of distinct biological phenotypes in patients with acute respiratory distress syndrome by cluster analysis. Thorax. 2017;72(10):876-883.

31. Fenner JE, et al. Suppressor of cytokine signaling 1 regulates the immune response to infection by a unique inhibition of type I interferon activity. Nat Immunol. 2006;7(1):33-39.

32. Alexander WS, et al. SOCS1 is a critical inhibitor of interferon gamma signaling and prevents the potentially fatal neonatal actions of this cytokine. Cell. 1999;98(5):597-608

33. Lew DJ, Decker T, Strehlow I, Darnell JE. Overlapping elements in the guanylate-binding protein gene promoter mediate transcriptional induction by alpha and gamma interferons. Mol Cell Biol. 1991;11(1):182-191.

34. Kim BH, Shenoy AR, Kumar P, Das R, Tiwari S, MacMicking JD. A family of IFN- $\gamma$-inducible $65-\mathrm{kD}$ GTPases protects against bacterial infection. Science. 2011;332(6030):717-721.

35. Satoh J, Takitani M, Miyoshi J, Kino Y. RNA-Seq data analysis identifies the comprehensive profile ofin vivo interferon- $\beta$-stimulated genes in multiple sclerosis. Clin Exp Neuroimmunol. 2016;7(1):39-51.

36. Sasai M, et al. Essential role for GABARAP autophagy proteins in interferon-inducible GTPase-mediated host defense. Nat Immunol. 2017;18(8):899-910.

37. Boulet I, et al. Lipopolysaccharide- and interferon-gamma-induced expression of hck and lyn tyrosine kinases in murine bone marrow-derived macrophages. Oncogene. 1992;7(4):703-710.

38. Finak G, et al. MAST: a flexible statistical framework for assessing transcriptional changes and characterizing heterogeneity in single-cell RNA sequencing data. Genome Biol. 2015;16:278.

39. Jiang C, et al. Inactivation of Rab11a GTPase in Macrophages Facilitates Phagocytosis of Apoptotic Neutrophils. J Immunol. 2017;198(4):1660-1672.

40. Pande J, Mallhi KK, Sawh A, Szewczyk MM, Simpson F, Grover AK. Aortic smooth muscle and endothelial plasma membrane $\mathrm{Ca} 2+$ pump isoforms are inhibited differently by the extracellular inhibitor caloxin 1b1. Am J Physiol, Cell Physiol. 2006;290(5):C1341-C1349.

41. Millar FR, Summers C, Griffiths MJ, Toshner MR, Proudfoot AG. The pulmonary endothelium in acute respiratory distress syndrome: insights and therapeutic opportunities. Thorax. 2016;71(5):462-473.

42. Rentz TJ, Poobalarahi F, Bornstein P, Sage EH, Bradshaw AD. SPARC regulates processing of procollagen I and collagen fibrillogenesis in dermal fibroblasts. J Biol Chem. 2007;282(30):22062-22071.

43. Sangaletti S, et al. SPARC Is a New Myeloid-Derived Suppressor Cell Marker Licensing Suppressive Activities. Front Immunol. 2019;10:1369.

44. Uhel F, et al. Early Expansion of Circulating Granulocytic Myeloid-derived Suppressor Cells Predicts Development of Nosoco- 
mial Infections in Patients with Sepsis. Am J Respir Crit Care Med. 2017;196(3):315-327.

45. Kamo T, et al. Prognostic values of the Berlin definition criteria, blood lactate level, and fibroproliferative changes on highresolution computed tomography in ARDS patients. BMC Pulm Med. 2019;19(1):37.

46. Kitsios GD, et al. Host-Response Subphenotypes Offer Prognostic Enrichment in Patients With or at Risk for Acute Respiratory Distress Syndrome. Crit Care Med. 2019;47(12):1724-1734.

47. Everitt AR, et al. IFITM3 restricts the morbidity and mortality associated with influenza. Nature. 2012;484(7395):519-523.

48. Hanna J, Bechtel P, Zhai Y, Youssef F, McLachlan K, Mandelboim O. Novel insights on human NK cells' immunological modalities revealed by gene expression profiling. J Immunol. 2004;173(11):6547-6563.

49. Chen Y, Shi JX, Pan XF, Feng J, Zhao H. DNA microarray-based screening of differentially expressed genes related to acute lung injury and functional analysis. Eur Rev Med Pharmacol Sci. 2013;17(8):1044-1050.

50. Randolph GJ, Jakubzick C, Qu C. Antigen presentation by monocytes and monocyte-derived cells. Curr Opin Immunol. 2008;20(1):52-60.

51. Nick JA, et al. Extremes of Interferon-Stimulated Gene Expression Associate with Worse Outcomes in the Acute Respiratory Distress Syndrome. PLoS One. 2016;11(9):e0162490.

52. Gough DJ, Messina NL, Clarke CJ, Johnstone RW, Levy DE. Constitutive type I interferon modulates homeostatic balance through tonic signaling. Immunity. 2012;36(2):166-174.

53. Jiang Z, Chen Z, Li L, Zhou W, Zhu L. Lack of SOCS3 increases LPS-induced murine acute lung injury through modulation of Ly6C(+) macrophages. Respir Res. 2017;18(1):217.

54. Messmer-Blust AF, Balasubramanian S, Gorbacheva VY, Jeyaratnam JA, Vestal DJ. The interferon-gamma-induced murine guanylate-binding protein-2 inhibits rac activation during cell spreading on fibronectin and after platelet-derived growth factor treatment: role for phosphatidylinositol 3-kinase. Mol Biol Cell. 2010;21(14):2514-2528.

55. Boekhoudt GH, McGrath AG, Swisher JF, Feldman GM. Immune complexes suppress IFN- $\gamma$-induced responses in monocytes by activating discrete members of the SRC kinase family. J Immunol. 2015;194(3):983-989.

56. Qin Y, et al. MiR-125a Is a critical modulator for neutrophil development. PLoS Genet. 2017;13(10):e1007027.

57. Rolls A, et al. Sleep disruption impairs haematopoietic stem cell transplantation in mice. Nat Commun. 2015;6:8516.

58. Mohan R, et al. Differentially Expressed MicroRNA-483 Confers Distinct Functions in Pancreatic $\beta$ - and $\alpha$-Cells. J Biol Chem. 2015;290(32):19955-19966.

59. He B, et al. SOCS-3 is frequently silenced by hypermethylation and suppresses cell growth in human lung cancer. Proc Natl Acad Sci USA. 2003;100(24):14133-14138.

60. Kim MH, et al. Suppressor of cytokine signaling (SOCS) genes are silenced by DNA hypermethylation and histone deacetylation and regulate response to radiotherapy in cervical cancer cells. PLoS One. 2015;10(4):e123133.

61. Murray PJ. Immune regulation by monocytes. Semin Immunol. 2018;35:12-18.

62. Auffray C, et al. Monitoring of blood vessels and tissues by a population of monocytes with patrolling behavior. Science. 2007;317(5838):666-670.

63. Li XC, Miyasaka M, Issekutz TB. Blood monocyte migration to acute lung inflammation involves both CD11/CD18 and very late activation antigen-4-dependent and independent pathways. J Immunol. 1998;161(11):6258-6264.

64. Yadav H, Kor DJ. Platelets in the pathogenesis of acute respiratory distress syndrome. Am J Physiol Lung Cell Mol Physiol. 2015;309(9):L915-L923.

65. Harris RA, Bowker-Kinley MM, Huang B, Wu P. Regulation of the activity of the pyruvate dehydrogenase complex. Adv Enzyme Regul. 2002;42:249-259.

66. De Backer D, Creteur J, Zhang H, Norrenberg M, Vincent JL. Lactate production by the lungs in acute lung injury. Am J Respir Crit Care Med. 1997;156(4 Pt 1):1099-1104.

67. Cox D, Lee DJ, Dale BM, Calafat J, Greenberg S. A Rab11-containing rapidly recycling compartment in macrophages that promotes phagocytosis. Proc Natl Acad Sci USA. 2000;97(2):680-685

68. Xu Y, et al. Single-cell RNA sequencing identifies diverse roles of epithelial cells in idiopathic pulmonary fibrosis. JCI Insight. 2016;1(20):e90558.

69. MacParland SA, et al. Single cell RNA sequencing of human liver reveals distinct intrahepatic macrophage populations. Nat Commun. 2018;9(1):4383.

70. Morrell ED, et al. Peripheral and Alveolar Cell Transcriptional Programs Are Distinct in Acute Respiratory Distress Syndrome. Am J Respir Crit Care Med. 2018;197(4):528-532.

71. Morrell ED, et al. Cytometry TOF identifies alveolar macrophage subtypes in acute respiratory distress syndrome. JCI Insight. 2018;3(10):99281.

72. Morrell ED, et al. Alveolar Macrophage Transcriptional Programs Are Associated with Outcomes in Acute Respiratory Distress Syndrome. Am J Respir Crit Care Med. 2019;200(6):732-741.

73. Reilly JP, Christie JD, Meyer NJ. Fifty Years of Research in ARDS. Genomic Contributions and Opportunities. Am J Respir Crit Care Med. 2017;196(9):1113-1121.

74. Morrell ED, et al. Genetic Variation in MAP3K1 Associates with Ventilator-Free Days in Acute Respiratory Distress Syndrome. Am J Respir Cell Mol Biol. 2018;58(1):117-125.

75. Reilly JP, et al. Plasma angiopoietin-2 as a potential causal marker in sepsis-associated ARDS development: evidence from Mendelian randomization and mediation analysis. Intensive Care Med. 2018;44(11):1849-1858.

76. Singer M, et al. The Third International Consensus Definitions for Sepsis and Septic Shock (Sepsis-3). JAMA. 2016;315(8):801-810.

77. Zheng GX, et al. Massively parallel digital transcriptional profiling of single cells. Nat Commun. 2017;8:14049.

78. Butler A, Hoffman P, Smibert P, Papalexi E, Satija R. Integrating single-cell transcriptomic data across different conditions, technologies, and species. Nat Biotechnol. 2018;36(5):411-420.

79. Bindea G, et al. ClueGO: a Cytoscape plug-in to decipher functionally grouped gene ontology and pathway annotation networks. Bioinformatics. 2009;25(8):1091-1093.

80. Aibar S, et al. SCENIC: single-cell regulatory network inference and clustering. Nat Methods. 2017;14(11):1083-1086. 